Bull. Korean Math. Soc. 52 (2015), No. 4, pp. 1365-1373

http://dx.doi.org/10.4134/BKMS.2015.52.4.1365

\title{
ON THE HOLONOMIZATION OF SEMIHOLONOMIC JETS
}

\author{
WŁodzimierz M. MiKulski
}

\begin{abstract}
We find all $\mathcal{F} \mathcal{M}_{m}$-natural operators $A$ transforming torsion free classical linear connections $\nabla$ on $m$-manifolds $M$ into base preserving fibred maps $A(\nabla): \bar{J}^{r} Y \rightarrow J^{r} Y$ for $\mathcal{F} \mathcal{M}_{m}$-objects $Y$ with bases $M$, where $\bar{J}^{r}, J^{r}$ are the semiholonomic and holonomic jet functors of order $r$ on the category $\mathcal{F} \mathcal{M}_{m}$ of fibred manifolds with $m$-dimensional bases and their fibred maps with embeddings as base maps.
\end{abstract}

\section{Introduction}

All manifolds considered in the paper are assumed to be finite dimensional, without boundaries, Hausdorff, second countable and smooth (of class $C^{\infty}$ ). Maps between manifolds are assumed to be of class $C^{\infty}$.

The classical theory of higher order jets was introduced by C. Ehresmann, [2]. For semiholonomic jets, we refer to the paper by P. Libermann, [8]. Higher order jets are a very powerful tool in differential geometry and in mathematical physics. For example, holonomic jets globalize the theory of differential systems and semiholonomic jets play an important role in the calculus of variations and in the theory of partial differential equations, [11], [12]. The theory of jets and connections forms the geometrical background for field theories and theoretical physics, [7], [9]. Holonomic and semiholonomic prolongation functors $J^{r}$ and $\bar{J}^{r}$ on the category $\mathcal{F M}_{m}$ of fibred manifolds with $m$-dimensional bases and their fibred maps with embeddings as base maps are classical examples of fiber product preserving bundle functors (i.e., bundle functors $F$ in the sense of [5] on $\mathcal{F} \mathcal{M}_{m}$ such that $F\left(Y_{1} \times_{M} Y_{2}\right)=F Y_{1} \times_{M} F Y_{2}$ for any $\mathcal{F} \mathcal{M}_{m}$-objects $Y_{1}$ and $Y_{2}$ with the same basis $M)$. The full description of fiber product preserving bundle functors $F$ on $\mathcal{F M}_{m}$ in terms of their corresponding triples $\left(A^{F}, H^{F}, t^{F}\right)$ can be found in [6] (see also [3]).

If $r=2$, we have the well-known symmetrization (holonomization) $\bar{J}^{2} Y \rightarrow$ $J^{2} Y$ of second order semiholonomic jets, i.e., we can produce canonically second order holonomic jets from second order semiholonomic ones. In [1], M.

Received October 25, 2014; Revised December 31, 2014.

2010 Mathematics Subject Classification. 58A05, 58A20, 58A32.

Key words and phrases. fiber product preserving bundle functor, natural operator, classical linear connection, semiholonomic jet, holonomic jet. 
Doupovec and the author proved that for $r \geq 3$ and $m \geq 2$ there is no symmetrization (holonomization) $\bar{J}^{r} Y \rightarrow J^{r} Y$, i.e., we cannot produce canonically $r$-order holonomic jets from $r$-order semiholonomic ones. So, to obtain a symmetrization $\bar{J}^{r} Y \rightarrow J^{r} Y$ (for $r \geq 3$ and $m \geq 2$ ), the using of an auxiliary object is unavoidable. In [10], to produce a symmetrization $\bar{J}^{r} Y \rightarrow J^{r} Y$ (for $r \geq 3$ and $m \geq 2$ ), we used classical linear connection $\nabla$ on the base of $Y$. The symmetrization $\bar{J}^{r} Y \rightarrow J^{r} Y$ depending on torsion free classical linear connection $\nabla$ on the base of $Y$ can be interpreted as an $\mathcal{F} \mathcal{M}_{m}$-natural operator $S$ transforming torsion free classical linear connections $\nabla$ on $m$-manifolds $M$ into base preserving fibred maps $S(\nabla): \bar{J}^{r} Y \rightarrow J^{r} Y$ for $\mathcal{F} \mathcal{M}_{m}$-objects $Y$ with bases $M$.

In general, let $F^{1}, F^{2}$ be fiber product preserving bundle functors on $\mathcal{F} \mathcal{M}_{m}$ of order $r$ and $\left(A^{F^{1}}, H^{F^{1}}, t^{F^{1}}\right)$ and $\left(A^{F^{2}}, H^{F^{2}}, t^{F^{2}}\right)$ be their corresponding triples. An $\mathcal{F} \mathcal{M}_{m}$-natural operator $A$ (in the sense of [5]) transforming torsion free classical linear connections $\nabla$ on $m$-manifolds $M$ into base preserving fibred maps $A(\nabla): F^{1} Y \rightarrow F^{2} Y$ for $\mathcal{F} \mathcal{M}_{m}$-objects $Y$ with bases $M$ is an $\mathcal{F} \mathcal{M}_{m^{-}}$ invariant family $A$ of regular operators

$$
A: \underline{Q}^{\tau}(M) \rightarrow C_{M}^{\infty}\left(F^{1} Y, F^{2} Y\right)
$$

for $\mathcal{F} \mathcal{M}_{m}$-objects $Y$ with bases $M$, where $\underline{Q}^{\tau}(M)$ is the space of torsion free classical linear connections on $M$ and $C_{M}^{\infty}\left(\overline{F^{1}} Y, F^{2} Y\right)$ is the space of base preserving fibred maps $F^{1} Y \rightarrow F^{2} Y$. The $\mathcal{F} \mathcal{M}_{m}$-invariance of $A$ means that if $f: Y^{1} \rightarrow Y^{2}$ is an $\mathcal{F} \mathcal{M}_{m}$-map with the base map $f: M^{1} \rightarrow M^{2}$ and $\nabla^{1} \in Q^{\tau}\left(M^{1}\right)$ and $\nabla^{2} \in Q^{\tau}\left(M^{2}\right)$ are $f$-related, then $A\left(\bar{\nabla}^{1}\right)$ and $A\left(\nabla^{2}\right)$ are $f$ related (i.e., $\left.F^{2} f \circ A\left(\nabla^{1}\right)=A\left(\nabla^{2}\right) \circ \bar{F}^{1} f\right)$. The regularity of $A$ means that $A$ transforms smoothly parametrized families of torsion free classical linear connections into smoothly parametrized families of fibred maps.

In [10], we studied the existence problem of $\mathcal{F} \mathcal{M}_{m}$-natural operators $A$ transforming torsion free classical linear connections $\nabla$ on $m$-manifolds $M$ into base preserving fibred maps $A(\nabla): F^{1} Y \rightarrow F^{2} Y$ for $\mathcal{F} \mathcal{M}_{m}$-objects $Y$ with bases $M$. We characterized fiber product preserving bundle functors $F^{1}, F^{2}$ on $\mathcal{F} \mathcal{M}_{m}$ admitting such $\mathcal{F} \mathcal{M}_{m}$-natural operators $A$ by means of the existence of (socalled) quasi morphisms of admissible triples. Next, in [4], I. Kolář extended this result to the existence problem of natural maps depending on reduction of frame bundles.

In the present paper, we study the classification problem of $\mathcal{F} \mathcal{M}_{m}$-natural operators $A$ transforming torsion free classical linear connections $\nabla$ on $m$ manifolds $M$ into base preserving fibred maps $A(\nabla): F^{1} Y \rightarrow F^{2} Y$ for $\mathcal{F} \mathcal{M}_{m^{-}}$ objects $Y$ with bases $M$, where $F^{1}, F^{2}$ are fiber product preserving bundle functors of order $r$ on $\mathcal{F} \mathcal{M}_{m}$ with their triples $\left(A^{F^{1}}, H^{F^{1}}, t^{F^{1}}\right)$ and $\left(A^{F^{2}}, H^{F^{2}}, t^{F^{2}}\right)$ respectively. We deduce that any such $A$ is of finite order and that there is the bijection between the space of such natural operators $A$ of finite order $q \geq r$ and the space of $G L(m)$-invariant maps $\Phi^{A}: Q^{q} \times A^{F^{1}} \rightarrow A^{F^{2}}$ such 
that $\mu_{\xi}: A^{F^{1}} \rightarrow A^{F^{2}}$ is an algebra homomorphism with $t^{F^{2}}=\mu_{\xi} \circ t^{F^{1}}$ for any $\xi \in Q^{q}$, where $Q^{q}$ is defined in Item 1 and $\mu_{\xi}(v):=\Phi^{A}(\xi, v), v \in A^{F^{1}}$ (see, Theorem 1). As an application, we describe all $\mathcal{F} \mathcal{M}_{m}$-natural operators $A$ transforming torsion free classical linear connections $\nabla$ on $m$-manifolds $M$ into base preserving fibred maps $A(\nabla): \bar{J}^{r} Y \rightarrow J^{r} Y$ for $\mathcal{F} \mathcal{M}_{m}$-objects $Y$ with bases $M$, where $\bar{J}^{r}, J^{r}$ are the semiholonomic and holonomic jet functors of order $r$ on $\mathcal{F} \mathcal{M}_{m}$, respectively.

\section{Invariant maps from natural operators}

Given a non negative integer $q$, let $Q^{q}$ be the (vector) space of $q$-jets at $0 \in \mathbb{R}^{m}$ of torsion free classical linear connections $\nabla=\left(\nabla_{j k}^{i}\right)$ on $\mathbb{R}^{m}\left(\nabla_{j k}^{i}\right.$ are the Christofell symbols of $\nabla$ in the identity map) such that

$$
\sum_{j, k=1}^{m} \nabla_{j k}^{i}(x) x^{j} x^{k}=0, i=1, \ldots, m .
$$

The condition (1) means that the identity map $i d_{\mathbb{R}^{m}}$ is $\nabla$-normal with center 0 (and vice-versa).

Let $F^{1}$ and $F^{2}$ be fiber product preserving bundle functors on $\mathcal{F} \mathcal{M}_{m}$ of order $r$ and $\left(A^{F^{1}}, H^{F^{1}}, t^{F^{1}}\right)$ and $\left(A^{F^{2}}, H^{F^{2}}, t^{F^{2}}\right)$ be their triples.

Lemma 1. Let $A$ be an $\mathcal{F M}_{m}$-natural operator of finite order $q \geq r$ transforming torsion free classical linear connections $\nabla$ on $m$-manifolds $M$ into base preserving fibred maps $A(\nabla): F^{1} Y \rightarrow F^{2} Y$ for $\mathcal{F} \mathcal{M}_{m}$-objects $Y$ with bases $M$.

(i) Given $\xi=j_{0}^{q} \nabla \in Q^{q}$, a map $\mu_{\xi}: A^{F^{1}} \rightarrow A^{F^{2}}, \mu_{\xi}(v):=A(\nabla)(v)$, $v \in A^{F^{1}}=F_{0}^{1}\left(\mathbb{R}^{m} \times \mathbb{R}\right)$ is a (well-defined because of the order argument) homomorphism of Weil algebras and $\mu_{\xi} \circ t^{F^{1}}=t^{F^{2}}$.

(ii) The resulting map $\Phi^{A}: Q^{q} \times A^{F^{1}} \rightarrow A^{F^{2}}, \Phi^{A}(\xi, v):=\mu_{\xi}(v)$ is $G L(m)$ invariant, i.e., it satisfies the condition

$$
\Phi^{A}\left(B . \xi, H^{F^{1}}(B)(v)\right)=H^{F^{2}}(B)\left(\Phi^{A}(\xi, v)\right)
$$

for any $B \in G L(m), v \in A^{F^{1}}$ and $\xi=j_{0}^{q} \nabla \in Q^{q}$, where $B . \xi=j_{0}^{q}\left(B_{*} \nabla\right)$ is the usual action.

Proof. Let $\tilde{F}^{1}, \tilde{F}^{2}$ be bundle functors on $\mathcal{M} f$ (=the category of manifolds and maps) given by

$$
\tilde{F}^{i} N=F_{0}^{i}\left(\mathbb{R}^{m} \times N\right) \text { and } \tilde{F}^{i} f=F^{i}\left(i d_{\mathbb{R}^{m}} \times f\right)_{\mid \tilde{F}^{i} N}: \tilde{F}^{i} N \rightarrow \tilde{F}^{i} N_{1}
$$

for any manifold $N$ and any map $f: N \rightarrow N_{1}$. They are product preserving (as $F^{i}$ are fiber product preserving) and $A^{F^{i}}$ are their Weil algebras (as $A^{F^{i}}=$ $\left.\tilde{F}^{i} \mathbb{R}\right)$. Given a torsion free classical linear connection $\nabla$ on $\mathbb{R}^{m}$, the family $\nu$ of maps $\nu_{N}: \tilde{F}^{1} N \rightarrow \tilde{F}^{2} N, \nu_{N}:=A(\nabla)_{\mid \tilde{F}^{1} N}$ for manifolds $N$ is a natural transformation $\tilde{F}^{1} \rightarrow \tilde{F}^{2}$ of product preserving bundle functors. Then $\mu_{\xi}$ : $A^{F^{1}} \rightarrow A^{F^{2}}$ is an algebra homomorphism (as $\left.\mu_{\xi}=\nu_{\mathbb{R}}\right)$. 
For any $f: \mathbb{R}^{m} \rightarrow \mathbb{R}$ we have

$$
\begin{aligned}
\mu_{\xi}\left(t^{F^{1}}\left(j_{0}^{r} f\right)\right) & =A(\nabla)\left(F_{0}^{1}\left(i d_{\mathbb{R}^{m}}, f\right)\left(\theta^{F^{1}}\right)\right) \\
& =F_{0}^{2}\left(i d_{\mathbb{R}^{m}}, f\right)\left(A(\nabla)\left(\theta^{F^{1}}\right)\right) \\
& =F_{0}^{2}\left(i d_{\mathbb{R}^{m}}, f\right)\left(\theta^{F^{2}}\right) \\
& =t^{F^{2}}\left(j_{0}^{r} f\right),
\end{aligned}
$$

where $\theta^{F^{i}}$ is the unique point from $F_{0}^{i} \mathbb{R}^{m}$.

By the invariance of $A$ with respect to $B \times i d_{\mathbb{R}}$, we have

$$
\begin{aligned}
\Phi^{A}\left(B . \xi, H^{F^{1}}(B)(v)\right) & =A\left(B_{*} \nabla\right)\left(F^{1}\left(B \times i d_{\mathbb{R}}\right)(v)\right) \\
& =F^{2}\left(B \times i d_{\mathbb{R}}\right)(A(\nabla)(v)) \\
& =H^{F^{2}}(B)\left(\Phi^{A}(\xi, v)\right)
\end{aligned}
$$

for any $B \in G L(m), v \in A^{F^{1}}$ and $\xi=j_{0}^{q} \nabla \in Q^{q}$.

\section{Natural operators from invariant maps}

Let $F^{1}, F^{2},\left(A^{F^{1}}, H^{F^{1}}, t^{F^{1}}\right)$ and $\left(A^{F^{2}}, H^{F^{2}}, t^{F^{2}}\right)$ be as in Item 1.

Lemma 2. Let $q \geq r$. Suppose $\Phi: Q^{q} \times A^{F^{1}} \rightarrow A^{F^{2}}$ is a map such that for any $\xi \in Q^{q}$ the map $\mu_{\xi}: A^{F^{1}} \rightarrow A^{F^{2}}, \mu_{\xi}(v):=\Phi(\xi, v)$ is an algebra homomorphism with $\mu_{\xi} \circ t^{F^{1}}=t^{F^{2}}$, and suppose that $\Phi$ is $G L(m)$-invariant, i.e., $\Phi\left(B . \xi, H^{F^{1}}(B)(v)\right)=H^{F^{2}}(B)(\Phi(\xi, v))$ for any $B \in G L(m), \xi \in Q^{q}$ and $v \in A^{F^{1}}$. Then there exists an $\mathcal{F M}_{m}$-natural operator $A^{\Phi}$ of order $q$ transforming torsion free classical linear connections $\nabla$ on $m$-manifolds $M$ into base preserving fibred maps $A^{\Phi}(\nabla): F^{1} Y \rightarrow F^{2} Y$ for $\mathcal{F M}_{m}$-objects $Y$ with bases $M$ satisfying $\Phi^{A^{\Phi}}=\Phi$.

If $A$ is an $\mathcal{F M}_{m}$-natural operator of order $q \geq r$ transforming torsion free classical linear connections $\nabla$ on $m$-manifolds $M$ into base preserving fibred maps $A(\nabla): F^{1} Y \rightarrow F^{2} Y$ for $\mathcal{F} \mathcal{M}_{m}$-objects $Y$ with bases $M$, then $A^{\Phi^{A}}=A$.

Proof. Let $Y$ be an $\mathcal{F} \mathcal{M}_{m}$-object with the projection $p_{Y}: Y \rightarrow M$. According to the general theory of fiber product preserving bundle functors $([3],[6])$, we have

$$
F^{i} Y=\left\{\left\langle j_{0}^{r} \varphi, v\right\rangle \in P^{r}(M)\left[T^{A^{F^{i}}} Y, H^{F^{i}}\right] \mid t^{F^{i}}\left(j_{0}^{r} \varphi\right)=T^{A^{F^{i}}}\left(p_{Y}\right)(v)\right\} .
$$

Let $\nabla$ be a torsion free classical linear connection on $M$. We define $A^{\Phi}(\nabla)$ : $F^{1} Y \rightarrow F^{2} Y$ as follows.

Let $w \in F_{x}^{1} Y, x \in M$. Let $\varphi$ be a $\nabla$-normal coordinate system with center $x$. There exists a unique $v \in T^{A^{F^{1}}} Y$ with $w=\left\langle j_{0}^{r} \varphi^{-1}, v\right\rangle$. We put

$$
A^{\Phi}(\nabla)(w):=\left\langle j_{0}^{r} \varphi^{-1}, \mu_{\xi}(v)\right\rangle,
$$


where $\xi=j_{0}^{q}\left(\varphi_{*} \nabla\right)$ and $\mu_{\xi}: T^{A^{F^{1}}} Y \rightarrow T^{A^{F^{2}}} Y$ is the Weil extension of the algebra homomorphism $\mu_{\xi}: A^{F^{1}} \rightarrow A^{F^{2}}, \mu_{\xi}(v)=\Phi(\xi, v)$.

If $\psi$ is an another $\nabla$-normal coordinate system with center $x$, then $\psi=B \circ \varphi$ for a $B \in G L(m)$. Then $w=\left\langle j_{0}^{r} \psi^{-1}, H^{F^{1}}(B)(v)\right\rangle$, and then

$$
A^{\Phi}(\nabla)(w)=\left\langle j_{0}^{r} \psi^{-1}, \mu_{B . \xi}\left(H^{F^{1}}(B)(v)\right)\right\rangle .
$$

So, to prove that the definition of $A^{\Phi}(\nabla)(w)$ is independent of the choice of $\nabla$-normal coordinate systems with centers $x$, we have to observe that

$$
\left\langle j_{0}^{r} \psi^{-1}, \mu_{B . \xi}\left(H^{F^{1}}(B)(v)\right)\right\rangle=\left\langle j_{0}^{r} \varphi^{-1}, \mu_{\xi}(v)\right\rangle .
$$

But, from the $G L(m)$-invariance of $\Phi$ we have $\mu_{B . \xi}=H^{F^{2}}(B) \circ \mu_{\xi} \circ H^{F^{1}}\left(B^{-1}\right)$. Then

$$
\begin{aligned}
\left\langle j_{0}^{r} \psi^{-1}, \mu_{B . \xi}\left(H^{F^{1}}(B(v))\right\rangle\right. & =\left\langle j_{0}^{r} \varphi^{-1} \cdot j_{0}^{r} B^{-1}, H^{F^{2}}(B)\left(\mu_{\xi}(v)\right)\right\rangle \\
& =\left\langle j_{0}^{r} \varphi^{-1}, \mu_{\xi}(v)\right\rangle .
\end{aligned}
$$

That is why, $A^{\Phi}(\nabla)(w)$ is a well defined element from $P^{r}(M)\left[T^{A^{F^{2}}} Y\right]$.

To see that $A^{\Phi}(\nabla)(w) \in F^{2} Y$, we have to prove that

$$
t^{F^{2}}\left(j_{0}^{r} \varphi^{-1}\right)=T^{A^{F^{2}}}\left(p_{Y}\right)\left(\mu_{\xi}(v)\right) .
$$

But we have that $t^{F^{1}}\left(j_{0}^{r} \varphi^{-1}\right)=T^{A^{F^{1}}}\left(p_{Y}\right)(v)$ as $\left\langle j_{0}^{r} \varphi^{-1}, v\right\rangle \in F^{1} Y$. By the assumption on $\mu_{\xi}$ we have $t^{F^{2}}=\mu_{\xi} \circ t^{F^{1}}$. Then

$$
\begin{aligned}
t^{F^{2}}\left(j_{0}^{r} \varphi^{-1}\right) & =\mu_{\xi} \circ t^{F^{1}}\left(j_{0}^{r} \varphi^{-1}\right)=\mu_{\xi} \circ T^{A^{F^{1}}}\left(p_{Y}\right)(v) \\
& =T^{A^{F^{2}}}\left(p_{Y}\right)\left(\mu_{\xi}(v)\right) .
\end{aligned}
$$

That is why, $A^{\Phi}(\nabla): F^{1} Y \rightarrow F^{2} Y$ is a well-defined map.

$A^{\Phi}(\nabla)$ is smooth because the exponent map $\operatorname{Exp}_{\nabla}$ is smooth. $A^{\Phi}\left(\nabla_{t}\right)$ is smoothly parametrized if $\nabla_{t}$ is because $\operatorname{Exp}_{\nabla_{t}}$ is smoothly parametrized if $\nabla_{t}$ is.

Now, we prove that $A^{\Phi}$ is $\mathcal{F} \mathcal{M}_{m}$-invariant. For, let $f: Y \rightarrow Y^{1}$ be an $\mathcal{F} \mathcal{M}_{m^{-}}$ map with the underlying map $\underline{f}: M \rightarrow M^{1}$ and $\nabla$ and $\nabla^{1}$ be $\underline{f}$-related torsion free classical linear connections on $M$ and $M^{1}$. Let $w=\left\langle j_{0}^{\bar{r}} \varphi^{-1}, v\right\rangle \in F_{x}^{1} Y$, $x \in M$, where $\varphi$ is a $\nabla$-normal coordinate system with center $x$. We see that $\varphi \circ \underline{f}^{-1}$ is $\nabla^{1}$-normal with center $\underline{f}(x)$. Put $\xi:=j_{0}^{r}\left(\varphi_{*} \nabla\right)\left(=j_{0}^{r}\left(\left(\varphi \circ \underline{f}^{-1}\right)_{*} \nabla^{1}\right)\right)$. Then

$$
\begin{aligned}
F^{2} f\left(A^{\Phi}(\nabla)(w)\right) & =F^{2} f\left(A^{\Phi}(\nabla)\left(\left\langle j_{0}^{r} \varphi^{-1}, v\right\rangle\right)\right) \\
& =F^{2} f\left(\left\langle j_{0}^{r} \varphi^{-1}, \mu_{\xi}(v)\right\rangle\right) \\
& =\left\langle j_{0}^{r}\left(\underline{f} \circ \varphi^{-1}\right), T^{A^{F^{2}}} f\left(\mu_{\xi}(v)\right)\right\rangle \\
& =\left\langle j_{0}^{r}\left(\underline{f} \circ \varphi^{-1}, \mu_{\xi}\left(T^{A^{F^{1}}} f(v)\right)\right\rangle\right. \\
& =A^{\Phi}\left(\nabla^{1}\right)\left(\left\langle j_{0}^{r}\left(\underline{f} \circ \varphi^{-1}\right), T^{A^{F^{1}}} f(v)\right\rangle\right)
\end{aligned}
$$




$$
\begin{aligned}
& =A^{\Phi}\left(\nabla^{1}\right)\left(F^{1} f\left(\left\langle j_{0}^{r} \varphi^{-1}, v\right\rangle\right)\right) \\
& =A^{\Phi}\left(\nabla^{1}\right)\left(F^{1} f(w)\right) .
\end{aligned}
$$

That is why, $A^{\Phi}$ is $f$-invariant for any $\mathcal{F} \mathcal{M}_{m}$-map $f$, i.e., $A^{\Phi}$ is $\mathcal{F} \mathcal{M}_{m}$-invariant.

Clearly, $A^{\Phi}(\nabla)_{\mid F_{x}^{1} Y}$ depends on $j_{x}^{q} \nabla$. (It easily follows from the fact that $j_{0_{x}}^{q+2}\left(\left(\operatorname{Exp}_{\nabla}\right)_{x}\right)$ depends on $j_{x}^{q} \nabla$.) That is why, $A^{\Phi}$ is of order $q$.

Let $\left\langle j_{0}^{r} i d_{\mathbb{R}^{m}}, v\right\rangle \in A^{F^{1}}=F_{0}^{1}\left(\mathbb{R}^{m} \times \mathbb{R}\right)$, where $v \in T^{A^{F^{1}}}\left(\mathbb{R}^{m} \times \mathbb{R}\right)$ with $t^{F^{1}}\left(j_{0}^{r} i d_{\mathbb{R}^{m}}\right)=T^{A^{F^{1}}}\left(p_{\mathbb{R}^{m} \times \mathbb{R}}\right)(v)$. Then $v=\left(t^{F^{1}}\left(j_{0}^{r} i d_{\mathbb{R}^{m}}\right), v_{1}\right) \in T^{A^{F^{1}}}\left(\mathbb{R}^{m} \times\right.$ $\mathbb{R})=T^{A^{F^{1}}} \mathbb{R}^{m} \times A^{F^{1}}$ with $v_{1} \in A^{F^{1}}$, and the identification $A^{F^{1}}=F_{0}^{1}\left(\mathbb{R}^{m} \times \mathbb{R}\right)$ is by $\left\langle j_{0}^{r} i d_{\mathbb{R}^{m}}, v\right\rangle=v_{1}$. We have the similar identification $A^{F^{2}}=F_{0}^{2}\left(\mathbb{R}^{m} \times \mathbb{R}\right)$, too. Under these identifications, the formula

$$
\Phi^{A^{\Phi}}(\xi, v)=\Phi(\xi, v)
$$

for $\xi \in Q^{q}$ and $v \in A^{F^{1}}$ is a immediate consequence of the formula

$$
A^{\Phi}(\nabla)\left(\left\langle j_{0}^{r} i d_{\mathbb{R}^{m}}, v\right\rangle\right):=\left\langle j_{0}^{r} i d_{\mathbb{R}^{m}}, \mu_{\xi}(v)\right\rangle
$$

for $\xi=j_{0}^{q} \nabla \in Q^{q}$ and $v \in T^{A^{F^{1}}}\left(\mathbb{R}^{m} \times \mathbb{R}\right)$ with $t^{F^{1}}\left(j_{0}^{r} i d_{\mathbb{R}^{m}}\right)=T^{A^{F^{1}}}\left(p_{\mathbb{R}^{m} \times \mathbb{R}}\right)(v)$.

Now, consider an $\mathcal{F M}_{m}$-natural operator $A$ of finite order $q \geq r$ transforming torsion free classical linear connections $\nabla$ on $m$-manifolds $M$ into base preserving fibred maps $A(\nabla): F^{1} Y \rightarrow F^{2} Y$ for $\mathcal{F M}_{m}$-objects with bases $M$. From the formula $\Phi^{A}(\xi, v)=A(\nabla)(v)$ for $\xi=j_{0}^{q} \nabla$ and $v \in A^{F^{1}}=F_{0}^{1}\left(\mathbb{R}^{m} \times \mathbb{R}\right)$ and the definition of $A^{\Phi^{A}}$ it follows that

$$
A^{\Phi^{A}}(\nabla)(v)=A(\nabla)(v)
$$

for any torsion free classical linear connection $\nabla$ on $\mathbb{R}^{m}$ with $i d_{\mathbb{R}^{m}}$ being $\nabla$ normal with center 0 and any $v \in F_{0}^{1}\left(\mathbb{R}^{m} \times \mathbb{R}\right)$. From the invariance of $A^{\Phi^{A}}$ and $A$ with respect to $\left(x^{1}, \ldots, x^{m}, t^{1} y^{1}, \ldots, t^{n} y^{n}\right)$ and the permutation of fibred coordinates it follows that

$$
A^{\Phi^{A}}(\nabla)\left(v^{1}, \ldots, v^{n}\right)=\left(A^{\Phi^{A}}(\nabla)\left(v^{1}\right), \ldots, A^{\Phi^{A}}(\nabla)\left(v^{n}\right)\right)
$$

and

$$
A(\nabla)\left(v^{1}, \ldots, v^{n}\right)=\left(A(\nabla)\left(v^{1}\right), \ldots, A(\nabla)\left(v^{n}\right)\right)
$$

for any torsion free classical linear connection $\nabla$ on $\mathbb{R}^{m}$ and any $v=\left(v^{1}, \ldots, v^{n}\right)$ $\in F_{0}^{1}\left(\mathbb{R}^{m} \times \mathbb{R}^{n}\right)=F_{0}^{1}\left(\mathbb{R}^{m} \times \mathbb{R}\right) \times \cdots \times F_{0}^{1}\left(\mathbb{R}^{m} \times \mathbb{R}\right)$. Then (2) holds for any $v \in F_{0}^{1}\left(\mathbb{R}^{m} \times \mathbb{R}^{n}\right)$ and any torsion free classical linear connection $\nabla$ on $\mathbb{R}^{m}$ with $i d_{\mathbb{R}^{m}}$ being $\nabla$-normal with center 0 . Then from the $\mathcal{F} \mathcal{M}_{m}$-invariance of $A^{\Phi^{A}}$ and $A$ it follows that $A^{\Phi^{A}}(\nabla)(v)=A(\nabla)(v)$ for any torsion free classical linear connection $\nabla$ on $M$ and any $v \in F_{x}^{1} Y, x \in M$, i.e., $A^{\Phi^{A}}=A$. 


\section{Finite orders of natural operators}

Let $F^{1}$ and $F^{2}$ be as in Item 1 .

Because of finite order theorems from [5], we have easily the following lemma.

Lemma 3. Any $\mathcal{F} \mathcal{M}_{m}$-natural operator A transforming torsion free classical linear connections $\nabla$ on $m$-manifolds $M$ into base preserving fibred maps $A(\nabla)$ : $F^{1} Y \rightarrow F^{2} Y$ for $\mathcal{F} \mathcal{M}_{m}$-objects $Y$ with bases $M$ is of finite order.

Schema of the proof. Because of finite order theorems ([5]), there exists a finite number $q$ such that

$$
j_{0}^{q} \nabla^{1}=j_{0}^{q} \nabla^{2} \Rightarrow A\left(\nabla^{1}\right)(v)=A\left(\nabla^{2}\right)(v)
$$

for any torsion free classical linear connections $\nabla^{1}$ and $\nabla^{2}$ respective close to $\nabla^{0}$ and any $v \in F_{0}^{1}\left(\mathbb{R}^{m} \times \mathbb{R}\right)$ respective close to the fiber homothety invariant element $c \in F_{0}\left(\mathbb{R}^{m} \times \mathbb{R}\right)$. On the other hand, given torsion free classical linear connections $\nabla^{1}$ and $\nabla^{2}$ on $\mathbb{R}^{m}$ and $v \in F_{0}^{1}\left(\mathbb{R}^{m} \times \mathbb{R}\right)$ we can find $s, t \in \mathbb{R} \backslash 0$ such that for $f=\left(t x^{1}, \ldots, t x^{m}, s y\right)$, the connections $f_{*} \nabla^{1}$ and $f_{*} \nabla^{2}$ are sufficiently close to $\nabla^{o}$ and $F^{1} f(v)$ is sufficiently close to $c$. Now, the lemma follows from the $\mathcal{F} \mathcal{M}_{m}$-invariance of $A$ and formula (3).

\section{The main result}

Summing up, we have the following theorem.

Theorem 1. Let $F^{1}, F^{2},\left(A^{F^{1}}, H^{F^{1}}, t^{F^{1}}\right),\left(A^{F^{2}}, H^{F^{2}}, t^{F^{2}}\right)$ and $Q^{q}$ be as in Item 1. There is the bijection between the space of $\mathcal{F} \mathcal{M}_{m}$-natural operators A of finite order $q \geq r$ transforming torsion free classical linear connections $\nabla$ on $m$-manifolds $M$ into base preserving fibred maps $A(\nabla): F^{1} Y \rightarrow F^{2} Y$ for $\mathcal{F} \mathcal{M}_{m}$-objects $Y$ with bases $M$ and the space of $G L(m)$-invariant maps $\Phi: Q^{q} \times A^{F^{1}} \rightarrow A^{F^{2}}$ such that $\mu_{\xi}: A^{F^{1}} \rightarrow A^{F^{2}}, \mu_{\xi}(v):=\Phi(\xi, v)$ is an algebra homomorphism with $\mu_{\xi} \circ t^{F^{1}}=t^{F^{2}}$ for any $\xi \in Q^{q}$. Moreover, any $\mathcal{F M}_{m}$ natural operator $A$ transforming torsion free classical linear connections $\nabla$ on $m$-manifolds $M$ into base preserving fibred maps $A(\nabla): F^{1} Y \rightarrow F^{2} Y$ for $\mathcal{F M}_{m}$-objects $Y$ with bases $M$ is of finite order.

\section{An application}

As an application of Theorem 1, we describe all $\mathcal{F} \mathcal{M}_{m}$-natural operators $A$ transforming torsion free classical linear connections $\nabla$ on $m$-manifolds $M$ into base preserving fibred maps $A(\nabla): \bar{J}^{r} Y \rightarrow J^{r} Y$ for $\mathcal{F} \mathcal{M}_{m}$-objects $Y$ with bases $M$, where $\bar{J}^{r}, J^{r}$ are the semiholonomic and holonomic jet functors of order $r$ on $\mathcal{F} \mathcal{M}_{m}$, respectively.

We know that $\bar{J}^{r}$ and $J^{r}$ are fiber product preserving bundle functors on $\mathcal{F} \mathcal{M}_{m}$ of order $r$. Let $\left(\bar{A}^{r}, \bar{H}^{r}, \bar{t}^{r}\right)$ and $\left(A^{r}, H^{r}, t^{r}\right)$ be the corresponding triples 
to $\bar{J}^{r}$ and $J^{r}$ respectively. Vector $G L(m)$-spaces $\bar{A}^{r}$ and $A^{r}$ (with respect to $\bar{H}_{\mid G L(m)}^{r}$ and $\left.H_{\mid G L(m)}^{r}\right)$ are of the form

$$
\bar{A}^{r}=\oplus_{k=0}^{r} \otimes^{k} \mathbb{R}^{m *} \text { and } A^{r}=\oplus_{k=0}^{r} S^{k} \mathbb{R}^{m *}
$$

with the standard tensor actions of $G L(m)$ (this is an easy observation, e.g. by the standard coordinate description), see [10]. Clearly, $t^{r}=i d$. The algebra multiplications of $\bar{A}^{r}$ and $A^{r}$ are given by a rather complicated formulas, which will not be used in the sequel. Clearly, the obvious inclusion $I: A^{r} \rightarrow \bar{A}^{r}$ is a morphism $I:\left(A^{r}, H^{r}, t^{r}\right) \rightarrow\left(\bar{A}^{r}, \bar{H}^{r}, \bar{t}^{r}\right)$ of triples (the one corresponding to the inclusion $J^{r} Y \subset \bar{J}^{r} Y$ ), see [10]. In particular, $\bar{t}^{r}=I$.

By Proposition 1 in [10], the usual symmetrization $s: \bar{A}^{r} \rightarrow A^{r}$ is a $G L(m)$ invariant algebra homomorphism such that $s \circ \bar{t}^{r}=t^{r}$. Thus we have the $G L(m)$-map $\Phi^{o}: Q^{q} \times \bar{A}^{r} \rightarrow A^{r}$ given by $\Phi^{o}(\xi, u)=s(u)$. Clearly, given $\xi \in Q^{q}$, the map $\mu_{\xi}: A^{r} \rightarrow A^{r}, \mu_{\xi}(v)=\Phi^{o}(\xi, v)$ is an algebra homomorphism with $\mu_{\xi} \circ \bar{t}^{r}=t^{r}$. So, we have the corresponding (symmetrization) $\mathcal{F M}_{m^{-}}$ natural operator $S$ transforming torsion free classical linear connections $\nabla$ on $m$-manifolds $M$ into base preserving fibred maps $S(\nabla): F^{1} Y \rightarrow F^{2} Y$ for $\mathcal{F M}_{m}$-objects $Y$ with bases $M$.

Lemma 4. Let $\Phi^{i}: Q^{q} \times \bar{A}^{r} \rightarrow A^{r}(i=1,2)$ be $G L(m)$-maps such that for any $\xi \in Q^{q}$ the maps $\mu_{\xi}^{i}: \bar{A}^{r} \rightarrow A^{r}, \mu_{\xi}^{i}(v)=\Phi^{i}(\xi, v)$ are algebra homomorphisms with $\mu_{\xi}^{i} \circ \bar{t}^{r}=t^{r}$. Then $\Phi^{1}=\Phi^{2}$.

Proof. We have to show that $\left\langle\Phi^{1}(\xi, u), w\right\rangle=\left\langle\Phi^{2}(\xi, u), w\right\rangle$ for any $\xi \in Q^{q}$, any $u \in \bar{A}^{r}$ and any $w \in S^{k} \mathbb{R}^{m}$ for $k=0, \ldots, r$. Because of the $G L(m)$ invariance of $\Phi^{1}$ and $\Phi^{2}$ we can assume that $w=\odot^{k} e_{1} \in S^{k} \mathbb{R}^{m}$, where $e_{1}=$ $(1,0, \ldots, 0) \in \mathbb{R}^{m}, k=0, \ldots, r$. Using the invariance of $\Phi^{1}$ and $\Phi^{2}$ with respect to $a_{t}: \mathbb{R}^{m} \rightarrow \mathbb{R}^{m}, a_{t}\left(x_{1}, \ldots, x_{m}\right)=\left(x_{1}, t x_{2}, \ldots, t x_{m}\right)$ for $t>0$ we obtain $\left\langle\Phi^{i}(\xi, u), w\right\rangle=\left\langle\Phi^{i}\left(a_{t} . \xi, a_{t} . u\right), w\right\rangle$ for $i=1,2$ and any $t>0$ (as $a_{t}$ preserves $\left.w=\odot^{k} e_{1}\right)$. Putting $t \rightarrow \infty$ we get $\left\langle\Phi^{i}(\xi, u), w\right\rangle=\left\langle\Phi^{i}\left(\xi^{o}, u^{o}\right), w\right\rangle$ for $i=1,2$, where $\xi^{o}=\lim _{t \rightarrow \infty}\left(a_{t} . \xi\right) \in Q^{q}$ and $u^{o}=\lim _{t \rightarrow \infty}\left(a_{t} . u\right) \in A^{r} \subset \bar{A}^{r}$ (these limits exist as $\left.\nabla_{11 ; 1 \cdots 1}^{i}=0\right)$. But $\Phi^{1}\left(\xi^{o}, u^{o}\right)=\Phi^{2}\left(\xi^{o}, u^{o}\right)$ because $\mu_{\xi^{o}}^{i}\left(u^{o}\right)=$ $\mu_{\xi}^{i} \circ I\left(u^{o}\right)=\mu_{\xi^{o}}^{i} \circ \bar{t}^{r}\left(u^{o}\right)=t^{r}\left(u^{o}\right)$ for $i=1,2$ as $u^{o} \in A^{r} \subset \bar{A}^{r}$. That is why, $\left\langle\Phi^{1}(\xi, u), w\right\rangle=\left\langle\Phi^{2}(\xi, u), w\right\rangle$.

Thus we have the following corollary of Theorem 1 and Lemma 4 .

Corollary 1. The symmetrization $\mathcal{F M}_{m}$-natural operator $S$ is the unique $\mathcal{F} \mathcal{M}_{m}$-natural operator A transforming torsion free classical linear connections $\nabla$ on $m$-manifolds $M$ into base preserving fibred maps $A(\nabla): \bar{J}^{r} Y \rightarrow J^{r} Y$ for $\mathcal{F} \mathcal{M}_{m}$-objects $Y$ with bases $M$.

The above corollary means that there is only one canonical procedure (called holonomization or symmetrization) to produce holonomic jets from semiholonomic ones by means of torsion free classical linear connections on bases. 


\section{References}

[1] M. Doupovec and W. M. Mikulski, Holonomic extension of connections and symmetrization of jets, Rep. Math. Phys. 60 (2007), no. 2, 299-316.

[2] C. Ehresmann, Extension du calcul des jets aux jets non holonomes, C. R. Acad. Sci. Paris 239 (1954), 1762-1764.

[3] I. Kolář, Weil bundles as generalized jet spaces, Handbook of global analysis, 625-664, 1214, Elsevier Sci. B. V., Amsterdam, 2008.

[4] N Natural maps depending on reduction of frame bundles, Ann. Polon. Math. 102 (2011), no. 1, 83-90.

[5] I. Kolár, P. W. Michor, and J. Slovák, Natural Operations in Differential Geometry, Springer-Verlag, Berlin 1993.

[6] I. Kolář and W. M. Mikulski, On the fiber product preserving bundle functors, Differential Geom. Appl. 11 (1999), no. 2, 105-111.

[7] M. de Leon and P. R. Rodrigues, Generalized Classical Mechanics and Field Theory, North-Holland Math. Studies 112, Amsterdam, 1985.

[8] P. Libermann, Introduction to the theory of semi-holonomic jets, Arch Math. (Brno) 33 (1996), 173-189.

[9] I. Mangiarotti and M. Modugno, Fibred spaces, jet spaces and connections for field theories, Proc. of Internat. Meeting "Geometry and Physics", Florence, 135-165, 1982, Pitagora Editrice, Bologna, 1983.

[10] W. M. Mikulski, On symmetrization of jets, Czechoslovak Math. J. 61(136) (2011), $157-168$.

[11] D. J. Saunders, The Geometry of Jet Bundles, London Math. Soc. Lecture Note Series 142, Cambridge Univ. Press, 1989.

[12] A. Vondra, Higher-order differential equations represented by connections on prolongations of a fibred manifold, Extracta Math. 15 (2000), no. 3, 421-512.

INSTITUTE OF MATHEMATICS

JAGIELLONIAN UNIVERSITY

UL S. LOJASIEWICZA 6

KRAKów, POLAND

E-mail address: Wlodzimierz.Mikulski@im.uj.edu.pl 